\title{
OBSTETRICAL PARALYSIS DUE TO ISCHAEMIA OF THE SPINAL CORD
}

\author{
BY \\ J. HUME ADAMS and HECTOR M. CAMERON* \\ From the Department of Pathology, the University and Western Infirmary, Glasgow, \\ and the Department of Pathology, Stobhill General Hospital, Glasgow
}

(RECEIVED FOR PUBLICATION MAY 1, 1964)

Obstetrical paralysis after a difficult labour is usually attributed to tearing or stretching of the nerve trunks of the brachial plexus or to avulsion of nerve roots from the spinal cord. Injury to the cord itself, however, is not excessively rare (Ford, 1960), the principal findings at necropsy being subdural or extradural haemorrhage, laceration or rupture of the cord, or a haematomyelia. In the case described here, paralysis of both arms at birth was found to be due to necrosis of the grey matter in the cervical part of the spinal cord, a condition that does not appear to have been described previously.

\section{Case Report}

Clinical History. The subject was a male infant whose birth was complicated by a face presentation. This was very easily converted to a vertex presentation by external version $4 \frac{1}{2}$ hours after the commencement of labour. The baby was then delivered without difficulty, the second stage lasting about 10 minutes. He was deeply cyanosed and responded to resuscitation only slowly. Both arms were flaccid and he was thought to have a bilateral Erb's palsy. In addition, it was noted that the head tended to flop forwards on to the chest. There was little change for a few days, but on the sixth day he was noted to be very pale and breathing rapidly. His colour improved when in an incubator but the respiration rate remained at about 100 a minute. Chest radiograph suggested that there might be some pulmonary collapse in the region of the lingula. On the next day, he became deeply cyanosed while being fed and the respirations became more rapid and strenuous. The diaphragm retracted with inspiration suggesting that it was paralysed, but this was not confirmed radiologically. The further chest radiographs showed that both lungs were consolidated and contained little air. The cerebrospinal fluid was normal, examination of the stools revealed no pathogenic organisms, and virological investigations were negative. The baby

* Present address: Department of Pathology, Royal Infirmary, Glasgow, C.4. continued to be cyanosed when out of oxygen and when being handled, and died on the tenth day.

Family History. There are two sibs: one, aged 4 years, is said to have suffered from haemorrhagic disease of the newborn, and is now very backward mentally; the other, aged 2 years, is of normal mentality but has gross asymmetry of the head, scoliosis, and weakness of the muscles on one side of the body.

Necropsy. A normally developed male infant, weighing $8 \mathrm{lb} .4 \mathrm{oz} .(3,741 \mathrm{~g}$.). The only abnormalities noted in the chest and abdomen were marked congestion and collapse of the lungs, and aspiration of material from the stomach into the trachea. The scalp and skull were normal. There was slight bruising of both leaves of the tentorium cerebelli, but no overt haemorrhage. The brain was normal externally and on section. The spinal cord in situ appeared entirely normal; there was no blood in the epidural space, and no tearing of nerve roots. Examination after removal revealed no evidence of haemorrhage or of external damage to the cord or nerve roots, and transverse cuts through the cervical, dorsal, and lumbar segments appeared normal. There was no indication of any traumatic damage to the vertebral column.

Before being examined in greater detail, the spinal cord was fixed in $10 \%$ formal saline for three weeks. Multiple transverse sections now revealed brownish discoloration and softening of the anterior horns extending from the second to the seventh cervical segments. All other levels appeared normal.

Histology. Relevant histological abnormalities were confined to the spinal cord where the principal feature was necrosis of the grey matter. From the second to the seventh cervical segments there was an almost total loss of nerve cells from the dorsal and ventral horns. The central parts of both ventral horns were rarefied and cystic (Fig. 1) but elsewhere there was increased cellularity of the grey matter caused by gemistocytic astrocytes (Fig. 2), hypertrophied microglial cells, and moderate capillary 


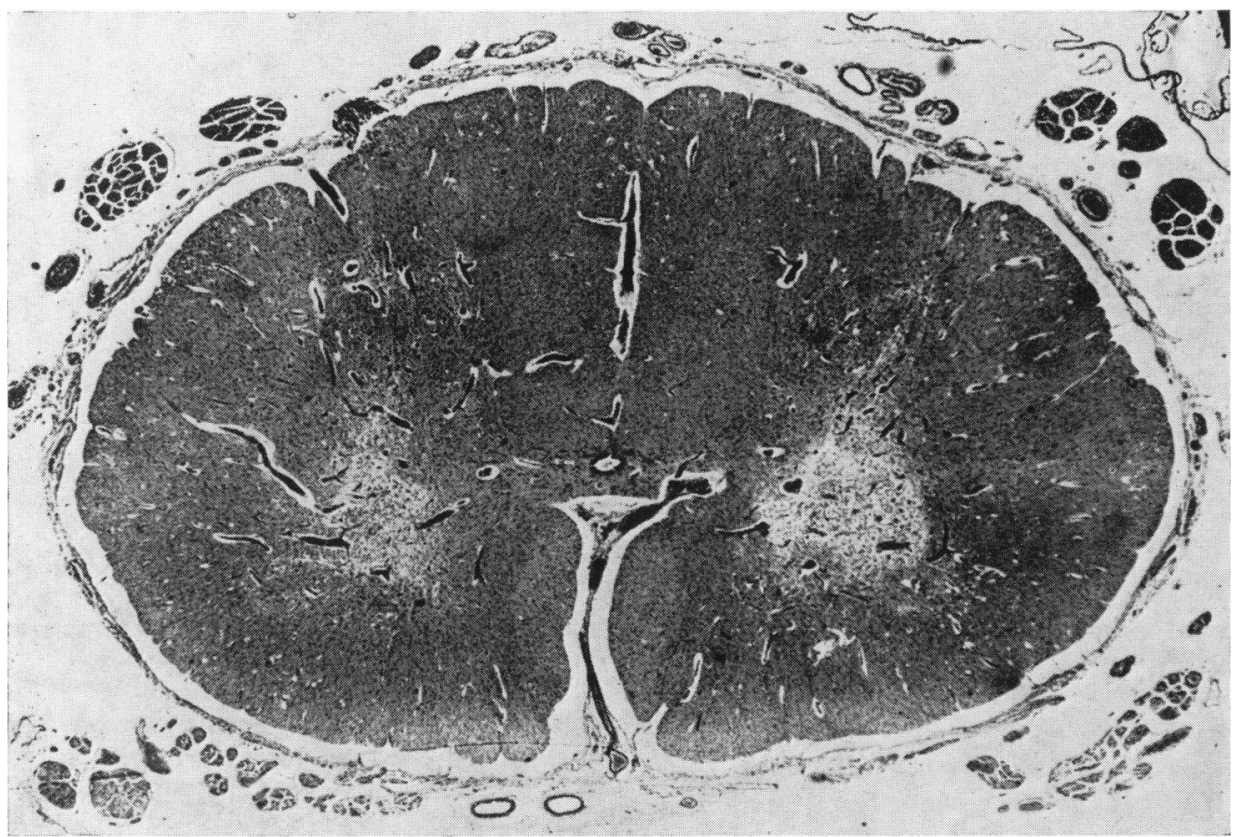

Fig. 1.-Cervical spinal cord (C.4 segment). Note the rarefaction and cystic change in the grey matter. (Haematoxylin and eosin.

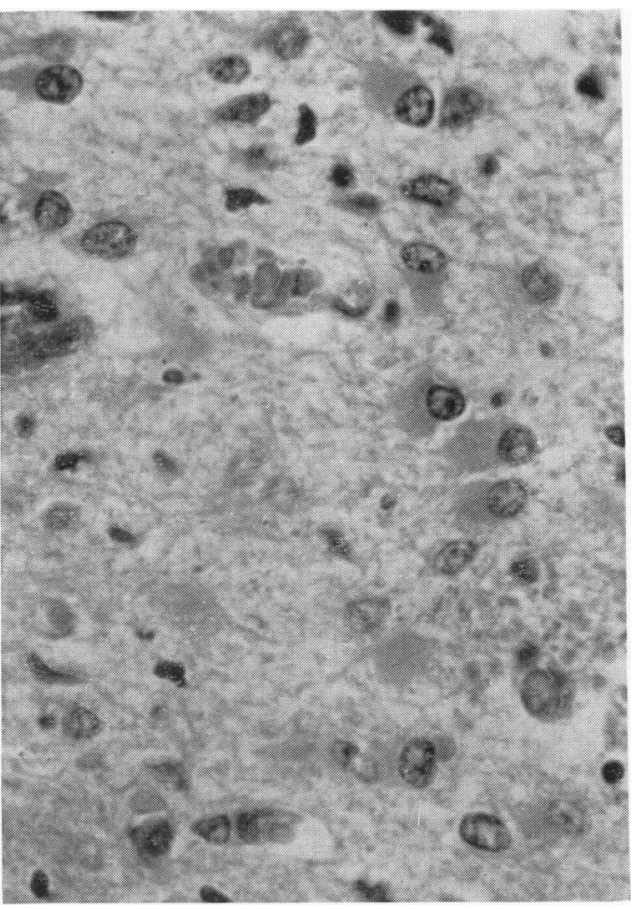

Fig. 2.-Same section as Fig. 1. Tip of dorsal grey horn. Note the numerous gemistocytic astrocytes and the absence of neurones. (Haematoxylin and eosin. $\times 500$.

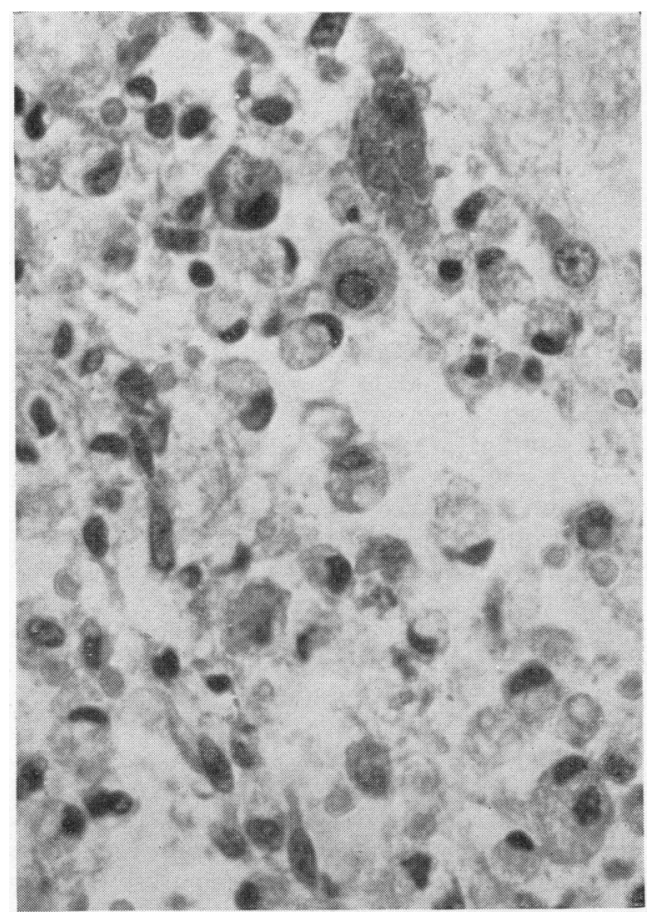

Fig. 3.-Same section as Fig. 1. Edge of cystic area in ventral grey horn. Note the numerous compound granular corpuscles. (Haematoxylin and eosin. $\times 500$.) 
endothelial hyperplasia. A very few large motor neurones could still be identified in the most anterior parts of the ventral horns. Adjacent to and within the cystic areas there were numerous compound granular corpuscles (Fig. 3) and many fragmented axis cylinders. In sections stained by Heidenhain's method for myelin and the Gros-Bielschowsky technique for axis cylinders, the white columns were normal but there was a fine fibrillary gliosis in the anterior and lateral white columns, most prominent adjacent to the grey matter, and in the grey matter. There was no infiltrate of inflammatory cells in the cord or in the leptomeninges, and in none of the numerous sections examined were there abnormal vessels or thrombi. The thoracic and lumbar segments of the cord were normal, and in sections stained by Marchi's method, no degenerating myelin was seen. Representative sections of cerebral hemispheres, cerebellum, and brain-stem were normal.

\section{Discussion}

The abnormalities found post mortem in this case indicate that the bilateral paralysis of the arms which existed from birth until death 10 days later was due to selective necrosis of the grey matter in the cervical part of the spinal cord. An infective aetiology, such as an intrauterine or neonatal poliomyelitis, can be excluded by the absence of a typical inflammatory infiltrate in the affected region or elsewhere in the central nervous system, by the normal cerebrospinal fluid and by the circumscribed nature of the lesion. Direct trauma, which might have been produced by the manipulation of the foetus, is unlikely to have produced such extensive necrosis without also having caused laceration or haemorrhage. In the absence of vascular occlusion or of a vascular malformation, we suggest that the necrosis of the spinal grey matter resulted from severe impairment of the local circulation.

There is a considerable amount of experimental evidence in support of this hypothesis, as a very similar lesion was observed in the cat after the circulation through an isolated segment of spinal cord had been drastically reduced by increasing the intradural pressure (Van Harreveld, 1941; Van Harreveld and Schadé, 1962). Further, in the cat, the dog, and the rabbit, reduction of the circulation through the spinal cord by occluding the aorta leads to selective necrosis of neurones in the central parts of the dorsal and ventral horns (Tureen, 1938; Krogh, 1945; Gelfan and Tarlov, 1959). The most peripheral neurones characteristically survive and the appearances of the cord a fortnight after the ischaemic episode are very similar to those observed in the present case. Symmetrical necrosis of the central neurones of the ventral horns of the spinal cord is present in some naturally occurring diseases in sheep and pigs (Innes and Saunders, 1962) and, in the absence of any other obvious aetiological factors, the authors conclude that impaired circulation through the cord is the main pathogenic factor.

In the case described here it seems likely that some mechanism had caused severe impairment of the circulation through the cervical part of the spinal cord of sufficient duration to cause selective necrosis of the grey matter. The only apparent factor is severe hyperextension of the neck associated with the face presentation early in labour. Hyperextension of the neck in adults with cervical spondylosis can obstruct the blood flow through the vertebral arteries (Yates and Hutchinson, 1961), but there is no evidence to suggest that a similar mechanism is of importance when there is no bony damage. Particular care was taken to ascertain if there was any damage to the vertebral canal in the present case, but none was found. Further, if both vertebral arteries had been obstructed by the hyperextension, some evidence of ischaemia in the brain-stem or cerebellum might have been expected.

There are, however, two other possible mechanisms: one is that anterior displacement of the ligamenta flava, which is known to occur in association with hyperextension of the neck (Taylor, 1953), had so compressed the spinal arteries and the cord that the circulation through it was grossly impaired; alternatively, hyperextension may itself have interfered with the blood flow through the spinal branches of the vertebral arteries. The former mechanism is analogous to the experimental procedure used by Van Harreveld and Schadé (1962) to produce a closely similar lesion in the spinal cord of the cat.

As the abnormalities in the spinal cord in the present case were not seen until it had been fixed, it seems possible that similar cases have been overlooked and that selective necrosis of spinal grey matter may be a hitherto unrecognized cause of obstetrical paralysis in the upper limbs.

\section{Summary}

A case of obstetrical paralysis which presented as a bilateral Erb's palsy was found at necropsy to be due to selective ischaemic necrosis of the grey matter in the cervical part of the spinal cord. It is suggested that this necrosis was caused by severe impairment of the circulation through the cord due to hyperextension of the neck.

We wish to thank Dr. E. G. Fox, Consultant Paediatrician, Stobhill Hospital, Glasgow, for permission to publish this case.

\section{REFERENCES}

Ford, F. R. (1960). Diseases of the Nervous System in Infancy, Childhood and Adolescence, 4th ed., Chapter 8. Thomas, Springfield, Illinois. 
Gelfan, S., and Tarlov, I. M. (1959). Interneurones and rigidity of spinal origin. J. Physiol. (Lond.), 146, 594.

Innes, J. R. M., and Saunders, L. Z. (1962). Comparative Neuropathology, Chapters 15 and 23. Academic Press, New York and London.

Krogh, E. (1945). Studies on the blood supply to certain regions in the lumbar part of the spinal cord. Acta physiol. scand., 10, 271.

Taylor, A. R. (1953). Mechanism and treatment of spinal-cord disorders associated with cervical spondylosis. Lancet, 1, 717.
Tureen, L. L. (1938). Circulation of the spinal cord and the effect of vascular occlusion. Res. Publ. Ass. nerv. ment. Dis., 18, 394.

Van Harreveld, A. (1941). The resistance of central synaptic conduction to asphyxiation. Amer. J. Physiol., 133, 572.

_ and Schadé, J. P. (1962). Nerve cell destruction by asphyxiation of the spinal cord. J. Neuropath. exp. Neurol., 21, 410.

Yates, P. O., and Hutchinson, E. C. (1961). Cerebral infarction: the role of stenosis of the extracranial cerebral arteries. Spec. Rep. Ser. med. Res. Coun. (Lond.), No. 300. 